\title{
Dificuldade visual em escolares: conhecimentos e ações de professores do ensino fundamental que atuam com alunos que apresentam visão subnormal
}

\author{
Students' visual difficulties:knowledge and actions of teachers of elementary schools \\ working with low vision students
}

\author{
Maria Elisabete R. Freire Gasparetto ${ }^{1}$ \\ Edméa Rita Temporini ${ }^{2}$ \\ Keila Miriam Monteiro de Carvalho ${ }^{3}$ \\ Newton Kara-José ${ }^{4}$
}

${ }_{1}^{1}$ Professora Doutora do Centro de Estudos e Pesquisas em Reabilitação "Prof. Dr. Gabriel Porto" - CEPRE Faculdade de Ciências Médicas da Universidade Estadual de Campinas.

${ }^{2}$ Professora Associada e Livre Docente em Metodologia de Pesquisa em Saúde na Disciplina de Oftalmologia da Faculdade de Ciências Médicas da Universidade Estadual de Campinas / Faculdade de Medicina da Universidade de São Paulo.

${ }^{3}$ Professora Assistente Doutora na Disciplina de Oftalmologia da Faculdade de Ciências Médicas da Universidade Estadual de Campinas.

${ }^{4}$ Professor Titular de Oftalmologia, Faculdade de Ciências Médicas da Universidade Estadual de Campinas e da Faculdade de Medicina da Universidade de São Paulo.

Contou com apoio financeiro da Secretaria de Educação Especial do Ministério da Educação: SEESP/MEC.

Endereço para correspondência: Maria Elisabete R.F Gasparetto, Rua Sampaio Vidal, 797 - Campinas (SP) CEP 13066-700

E-mail: gasparettoca@uol.com.br

Nota Editorial: Pela análise deste trabalho e por sua anuência na divulgação desta nota, agradecemos à Dra. Luciene Chaves Fernandes.

\begin{tabular}{|c|}
\hline RESUMO \\
\hline $\begin{array}{l}\text { Objetivos: 1) Verificar os conhecimentos e ações desenvolvidas por } \\
\text { professores do ensino fundamental, que atuam com alunos que apresen- } \\
\text { tam visão subnormal, em relação aos sinais e sintomas indicativos de } \\
\text { dificuldades visuais dos alunos; } 2 \text { ) Obter informações indispensáveis ao } \\
\text { planejamento de ações preventivas direcionadas à saúde ocular na escola. } \\
\text { Métodos: Realizou-se levantamento entre professores do ensino funda- } \\
\text { mental de escolas públicas do município de Campinas/SP, que atuavam } \\
\text { com alunos que apresentavam visão subnormal, no ano letivo de } 1999 \text {. } \\
\text { Foram incluídas } 23 \text { escolas onde se localizava essa população. Foi utilizado } \\
\text { questionário auto-aplicável como instrumento de coleta de dados. } \\
\text { Resultados: De } 84 \text { professores, } 68 \text { ( } 81 \% \text { ) responderam ao questionário. A } \\
\text { média de tempo de experiência profissional de magistério era de } 20,8 \text { anos. } \\
\text { A maioria (92,6\%) não relatou formação na área da deficiência visual. Em } \\
\text { relação ao conhecimento sobre os sinais e sintomas indicativos de dificul- } \\
\text { dade visual, a maioria indicou a dificuldade para ler na lousa ( } 94,1 \% \text {, } \\
\text { seguida da cefaléia ( } 89,7 \% \text { ) e a aproximação exagerada dos objetos aos } \\
\text { olhos ( } 88,2 \% \text { ). Desses professores, } 55,9 \% \text { identificaram alunos que apre- } \\
\text { sentavam dificuldades visuais. Entre os que declararam ter identificado } \\
\text { esses alunos, } 84,2 \% \text { proveram orientações ao escolar e } 63,2 \% \text { aos familia- } \\
\text { res para encaminhamento do problema. Somente } 26,3 \% \text { orientaramoaluno } \\
\text { a procurar o oftalmologista. Conclusão: Os professores apresentaram } \\
\text { conhecimento insuficiente quanto à saúde ocular e, portanto, as ações } \\
\text { desenvolvidas não foram completas e abrangentes. Sugere-se a implanta- } \\
\text { ção de um programa de saúde ocular em todo o sistema público de ensino, } \\
\text { visando desenvolver ações de prevenção da incapacidade visual, promo- } \\
\text { ção e recuperação da saúde ocular. }\end{array}$ \\
\hline
\end{tabular}

Descritores: Baixa visão; Transtornos da visão; Saúde escolar; Saúde ocular; Conhecimentos atitude e prática; Educação em saúde; Estudantes; Acuidade visual; Serviços de saúde escolar

\section{INTRODUÇÃOO}

A visão desempenha um papel predominante nos primeiros anos de vida, pois é um estímulo motivador para a comunicação e realização de ações. $\mathrm{O}$ relacionamento com o mundo exterior é realizado principalmente por meio da visão, de forma que os problemas oculares podem representar graves prejuízos para a aprendizagem e socialização das crianças ${ }^{(1)}$. Até a idade escolar, a 
deficiência visual pode passar despercebida pelos pais e familiares porque, no ambiente doméstico, a criança não tem noção que não enxerga bem, pois não exerce atividades que demandem esforço visual. Isso fica agravado, principalmente, devido à ausência de exames oftalmológicos periódicos ${ }^{(2)}$.

A deficiência visual mais acentuada e mais grave denominase baixa visão ou visão subnormal e pode ser definida como acuidade visual entre $6 / 18(0,3)$ e $3 / 60(0,05)$ com a melhor correção óptica no olho de melhor visão( ${ }^{(3)}$.

Para identificar a presença de dificuldade visual, KaraJosé, Almeida, Arieta, Araújo, Beggara e Oliveira sugerem a obrigatoriedade de exames oftalmológicos ou, pelo menos, da medida de acuidade visual, em crianças de quatro anos de idade (em média), por ocasião da matrícula na educação infantil ou do ingresso no ensino fundamental, quando a criança tem, em média, sete anos de idade ${ }^{(4)}$.

Estima-se que cerca de $20 \%$ de crianças em idade escolar apresentem dificuldades visuais devido a defeitos refracionais não corrigidos, estrabismo e ambliopia, entre outros. Em cada 1000 alunos do ensino fundamental, 100 são portadores de erros de refração, necessitando de óculos para a correção de hipermetropia, miopia e astigmatismo. Destes, aproximadamente 5\% apresentam redução de acuidade visual, isto é, menos de $50 \%$ da visão normal ${ }^{(5)}$.

Em pesquisa realizada sobre conhecimentos e práticas em saúde escolar com 1000 pessoas da cidade de Campinas - SP, verificou-se que apenas $24,4 \%$ dos informantes foram submetidos a exames oftalmológicos até os 7 anos de idade ${ }^{(6)}$. Em outro estudo, encontrou-se que $45,5 \%$ dos alunos de $1^{a}$ a $4^{a}$ série do ensino fundamental, que necessitavam de óculos, tiveram suas prescrições indicadas, pela primeira vez, por ocasião da referida pesquisa ${ }^{(7)}$.

Entende-se que toda criança deveria ser submetida a exame oftalmológico antes de seu ingresso na escola. Supõe-se que fatores socioeconômicos e culturais impeçam que isso ocorra, daí a importância da realização de programas de triagem da acuidade visual e detecção de problemas visuais na escola, preenchendo essa lacuna ${ }^{(8)}$.

A capacitação de profissionais para atuarem na promoção da saúde ocular e a adequada atenção primária em saúde, que inclui os programas para avaliação da acuidade visual e identificação de crianças com significativos erros de refração, são essenciais para o controle da deficiência visual na infância ${ }^{(9)}$.

Em relação à avaliação da acuidade visual, cabe ressaltar que ela vem sendo realizada nas escolas por meio do Teste de Snellen, utilizando-se o professor como examinador. Esta situação tem criado polêmica, gerando nos professores um descrédito em relação ao teste. Não se reconhece que a precisão do teste depende fundamentalmente de condições adequadas de aplicação e treinamento do examinador ${ }^{(10)}$, conforme foi observado em estudo sobre avaliação dos critérios de triagem visual de escolares de $1^{\mathrm{a}}$ série do ensino fundamental, mostrando que, $87,1 \%$ dos professores realizaram corretamente o teste de acuidade visual (todos os professores foram devidamente orientados, anteriormente $)^{(11)}$.
Sabe-se que muitos professores não concordam com a realização do teste da acuidade visual dos alunos, pois acreditam que esta seja função dos profissionais da área da saúde. Por isso, faz-se necessária a sensibilização destes profissionais, pois, em sala de aula, eles contam com uma situação ímpar em relação à observação das dificuldades visuais e queixas dos alunos nas diferentes atividades escolares.

No entanto, é necessário salientar que, nem sempre o aluno conseguirá verbalizar as dificuldades visuais que ocorrem com ele. Por este motivo, o professor deve ficar atento às possíveis manifestações como a dificuldade para se locomover, ler, copiar a matéria e desenhar, aproximando exageradamente os materiais dos olhos ${ }^{(12)}$.

Por isso, é imperativo que a escola participe de ações de promoção da saúde ocular, de identificação e encaminhamento de alunos a especialistas para diagnóstico e tratamento dos problemas visuais detectados. Percebe-se, contudo, que o pessoal de ensino, em geral precisa ser capacitado para conseguir realizar atividades de programas de prevenção da deficiência visual, na escola ${ }^{(13)}$.

Levando-se em conta essa problemática, foi realizada pesquisa com os objetivos de verificar os conhecimentos e ações desenvolvidas por professores do ensino fundamental em relação aos sinais e sintomas indicativos de dificuldades visuais de alunos e obter informações de modo a contribuir para o planejamento de ações preventivas direcionadas à saúde ocular na escola.

\section{MÉTODOS}

Foi realizado levantamento entre professores do ensino fundamental de escolas públicas municipais e estaduais do município de Campinas-SP que atuam com alunos que apresentam visão subnormal. Era condição sine qua non que o professor que constituísse a população tivesse experiência didática com o aluno que apresentasse visão subnormal, no ano considerado. Tratou-se de critério de inclusão, por supor conhecimentos sobre o assunto por parte do professor. Esta opção prendeu-se ao objeto de estudo e não à casuística.

No município de Campinas, durante o ano letivo de 1999, havia 84 professores que atendiam esses alunos, distribuídos em 13 escolas municipais e 11 escolas estaduais. A pesquisa cobriu, em média, uma sala de aula por escola. As salas de aula apresentavam a média de 34 alunos e a média de um aluno com visão subnormal por turma.

Foram incluídas neste estudo 12 (92,3\%) escolas municipais e 11 escolas estaduais, representando o total de escolas que aceitaram a realização da pesquisa. A população reduziu-se a 68 professores, ou seja, $81 \%$ da existente, em razão de afastamentos por licença médica, licença gestante e transferência de escola.

As escolas municipais foram selecionadas mediante indicação dos coordenadores do Programa de Educação Especial da Secretaria de Educação da Prefeitura Municipal de Campinas, por informações contidas no cadastro do Centro de Estu- 
dos e Pesquisas em Reabilitação "Prof. Dr. Gabriel Porto" (CEPRE) e no cadastro do Serviço de Visão Subnormal, ambos da Universidade Estadual de Campinas (UNICAMP). Para compor o grupo de escolas estaduais, o critério de inclusão baseou-se na presença de professores especializados em deficiência visual na unidade, pelo fato de que esses profissionais fornecem assistência aos alunos com visão subnormal. Além disso, obteve-se indicação de escolas estaduais por meio de serviços da comunidade e informações constante em registros dos serviços mencionados acima.

Foram investigadas as seguintes variáveis: idade do professor; tempo de experiência de magistério; formação especializada na área da deficiência visual; conhecimento de sinais e sintomas indicativos de dificuldade visual e ações em relação a escolares que apresentam dificuldade visual (Anexo 1).

Mediante estudo exploratório realizado com professores do ensino fundamental de escolas estaduais de municípios próximos a Campinas, elaborou-se questionário auto-aplicável como instrumento de coleta de dados, que foi aplicado aos professores no período de dezembro de 1999 a junho de 2000, tanto nas escolas municipais como nas escolas estaduais que incluem alunos com visão subnormal ${ }^{(14)}$.

O questionário foi entregue aos professores, juntamente com carta de instruções para o preenchimento na presença do pesquisador (Anexo 2). Após o preenchimento individual, o professor colocava o questionário em envelope, lacrando-o, assegurandose dessa forma anonimato e sigilo das informações.

\section{RESULTADOS}

O estudo foi composto por 68 professores (ambos os sexos) do ensino fundamental de escolas públicas municipais e estaduais do município de Campinas, com idade entre 25 e 64 anos. A média de idade foi de 44,2 anos. O tempo de experiência no magistério variou de 5 a 46 anos com média de 20,8 anos. Em relação à capacitação especializada, 92,6\% dos professores declararam não apresentar formação específica na área da deficiência visual, 4,4\% declararam ter realizado curso de especialização em educação especial, $1(1,5 \%)$ professor declarou ter realizado curso de extensão e $1(1,5 \%)$ declarou ter participado de treinamento eventual para atuar com deficientes visuais (Tabela 1).

Em relação ao conhecimento sobre os sinais e sintomas indicativos de dificuldade visual, todos os professores apresentaram respostas. A maioria $(94,1 \%)$ reconheceu a dificuldade para ler na lousa como causa de problema visual. Como se observa na tabela 2 , também é significativo o número de professores que indicam a cefaléia $(89,7 \%)$ e a aproximação exagerada dos objetos aos olhos $(88,2 \%)$ como causas de dificuldade visual. As opções menos apontadas dizem respeito ao estrabismo $(60,3 \%)$ e ao nistagmo $(54,4 \%)$. Quanto à detecção de escolares com dificuldades visuais, $38(55,9 \%)$ dos professores respondentes mencionaram ter realizado a identificação em sala de aula.
Os procedimentos desses 38 professores em relação aos alunos que apresentaram dificuldade visual estão indicados na tabela 3 , evidenciando que a maioria $(84,2 \%)$ informa ter orientado o aluno. Não obstante, é importante ressaltar que somente $26,3 \%$ dos professores declaram ter orientado o aluno a procurar o oftalmologista.

\begin{tabular}{|lrr|}
\hline $\begin{array}{l}\text { Tabela 1. Características profissionais de preparo e experiência } \\
\text { profissional para atuação junto a alunos portadores de baixa visão. } \\
\text { Professores do ensino fundamental. Campinas, SP, 1999 } \\
\text { n }\end{array}$ \\
$\begin{array}{lrr}\text { Características Profissionais (anos) } \\
\text { Tempo de exercício (anos) }\end{array}$ & f \\
$5-10$ & 9 & 13,2 \\
$11-20$ & 30 & 44,1 \\
$21-30$ & 20 & 29,5 \\
$31-46$ & 9 & 13,2 \\
& $x=20,8$ anos & \\
Preparação na área da deficiência visual & & \\
Nenhuma & 63 & 92,6 \\
Curso de especialização & 3 & 4,4 \\
Curso de extensão & 1 & 1,5 \\
Treinamento & 1 & 1,5 \\
\hline
\end{tabular}

Tabela 2. Conhecimentos sobre sinais e sintomas que podem indicar dificuldade visual do aluno. Professores do ensino fundamental. Campinas, SP, 1999

\begin{tabular}{lcc} 
& \multicolumn{3}{c}{$\mathrm{n}=68$} \\
Conhecimentos $^{*}$ & $f$ & $\%$ \\
Dificuldade para ler na lousa & 64 & 94,1 \\
Cefaléia & 61 & 89,7 \\
Aproximação exagerada dos objetos aos olhos & 60 & 88,2 \\
Franzir da testa & 56 & 82,4 \\
Desatenção ao andar & 52 & 76,5 \\
Desatenção em classe & 51 & 75,0 \\
Desinteresse por leitura & 50 & 73,5 \\
Lacrimejamento & 48 & 70,6 \\
Dificuldade em parear / discriminar cores & 46 & 67,6 \\
Fotofobia & 45 & 66,2 \\
Pender a cabeça durante a leitura & 42 & 61,8 \\
Estrabismo & 41 & 60,3 \\
Nistagmo & 37 & 54,4 \\
( $^{*}$ respostas múltiplas & & \\
\hline
\end{tabular}

Tabela 3. Ações em relação ao aluno que apresenta dificuldade visual. Professores do ensino fundamental. Campinas, SP, 1999

\begin{tabular}{lcc|} 
& \multicolumn{2}{c}{$\mathrm{n}=38$} \\
Ações $^{*}$ & $f$ & $\%$ \\
Orientou o aluno & 32 & 84,2 \\
Orientou pais / familiares & 24 & 63,2 \\
Comunicou a direção da escola & 24 & 63,2 \\
Orientou a procurar o oftalmologista & 10 & 26,3 \\
(*) respostas múltiplas & & \\
\end{tabular}




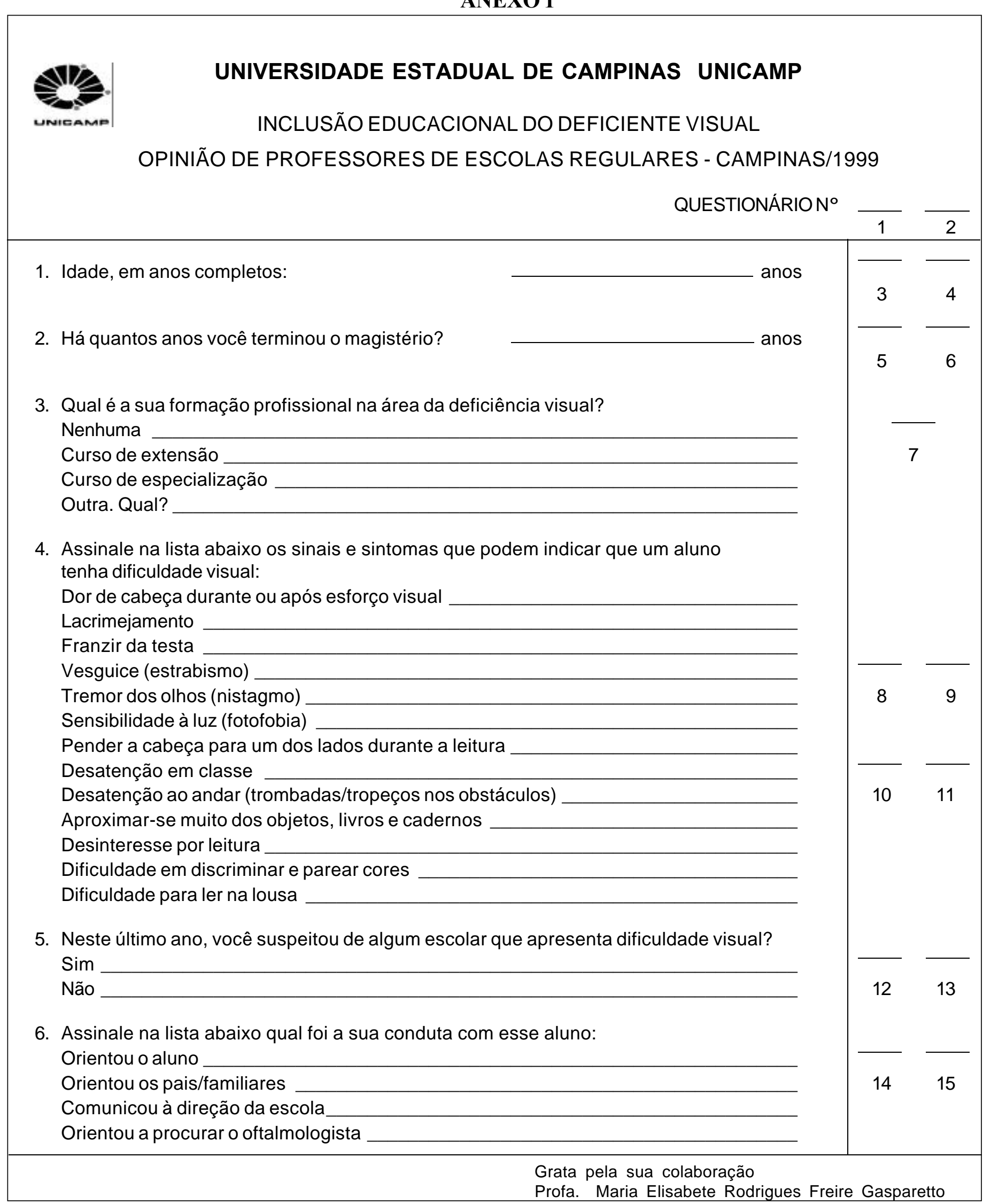


ANEXO 2

\title{
Campinas,... de..........de 1999.
}

\section{Prezado Professor:}

Com a presente, estamos Ihe entregando um questionário sobre a pesquisa "Opinião de Professores do Ensino Fundamental de Escolas Públicas Municipais e Estaduais de Campinas sobre a Inclusão Educacional de alunos que apresentam visão subnormal".

Esse estudo tem por finalidade subsidiar o planejamento de um programa de ações educativas referentes à inclusão educacional de alunos que tem visão subnormal.

Assim sendo, vimos solicitar sua preciosa colaboração no sentido de responder ao questionário anexo. Para tanto, solicitamos que observe as seguintes recomendações:

1. Leia com atenção, não só a pergunta, mas também todas as respostas.

2. Assinale com um $X$ a resposta que você quiser indicar.

3. Use caneta esferográfica.

4. Cada questão apresenta todas as respostas possíveis. Portanto não deixe nenhuma questão sem resposta.

5. Os números colocados ao lado do lugar destinado às respostas, assim como os que se encontram na coluna à direita, serão utilizados na codificação das questões e computação dos dados. Portanto, não os leve em consideração ao responder ao questionário.

\section{Para que esta pesquisa alcance elevado grau de validade e fidedignidade, é:}

Necessário que ela descreva realmente o que você faz e represente sua verdadeira opinião. E, para que você possa se expressar livremente, duas providências lhe são asseguradas:

1. O questionário não é assinado.

2. Respondido, o questionário deve ser colocado em envelope.

\author{
Cordiais cumprimentos \\ Maria Elisabete Rodrigues Freire Gasparetto
}

\section{DISCUSSÃO}

A análise dos resultados indica que os respondentes não são habilitados em deficiência visual, no entanto, declararam 20,8 anos como tempo médio de experiência profissional no magistério (Tabela 1). Pode-se supor que esse tempo de atuação tenha propiciado subsídios aos professores para suspeitarem das dificuldades visuais dos alunos, pois o contato diário no ambiente escolar possibilita conhecer o modo de ser de cada aluno e notar alterações na aparência ou na conduta. Nesse contexto ressalta-se a importância do professor no desenvolvimento de ações educativas de preservação e restauração do sistema visual de escolares ${ }^{(2)}$.

Em relação ao conhecimento de sinais e sintomas referentes à dificuldade visual, os professores apontaram com menor intensidade nistagmo, fotofobia e posicionamento de cabeça (Tabela 2). $\mathrm{O}$ fato de que estes sinais tenham sido menos indi- cados sugere desconhecimento a respeito da relação entre eles e a dificuldade visual.

O reconhecimento dos sinais pode ser realizado de duas formas: 1) por meio do comportamento do aluno, durante a realização de atividades em sala de aula como, por exemplo, a aproximação exagerada dos objetos, desatenção em classe, desinteresse por leitura entre outros; 2) por meio dos sinais visuais apresentados pelos alunos: estrabismo, nistagmo, lacrimejamento e o franzir da testa. A identificação de sintomas será alcançada na manifestação de queixas dos alunos como a cefaléia e a fotofobia ${ }^{(15)}$.

Se o professor consegue perceber os sinais por meio da observação das atividades escolares ou na aparência do aluno, esta percepção pode ser completada por meio de perguntas ao aluno a respeito dos sintomas (cefaléia, fotofobia), pois nem sempre este tipo de evidência é percebida ${ }^{(14)}$. Daí a importância do preparo do professor para realizar diagnóstico edu- 
cativo podendo, assim, auxiliar na prevenção de distúrbios ou agravos oftalmológicos ${ }^{(16)}$.

No período de 1973 a 1976, desenvolveu-se em São Paulo o Plano de Oftalmologia Sanitária Escolar (POSE) que tinha como objetivo detectar distúrbios visuais de escolares e prover a devida assistência, apoiado por atividades educativas. Dentre as atividades conduzidas no POSE, podem ser destacadas: observação pelo professor de sinais, sintomas e comportamentos do aluno que poderiam indicar problemas; identificação dos escolares com suspeita de distúrbios visuais e atuação do professor junto ao aluno e à família, no que se refere aos aspectos educacionais, bem como aos aspectos educativos relacionados à higiene visual. A partir de 1977, o POSE foi incorporado às Secretarias de Estado e da Educação e esse fato pode ter contribuído para a perda de continuidade das ações e conseqüente prejuízo dos escolares ${ }^{(1)}$.

Para preencher as lacunas existentes, o Conselho Brasileiro de Oftalmologia desenvolveu em 1998 a Campanha Nacional de Promoção da Saúde Ocular, com o atendimento de alunos da primeira série do ensino fundamental em escolas públicas, realizando a prescrição de óculos e também criando condições para facilitar a aquisição ou doação de óculos para os escolares ${ }^{(17)}$. No entanto, é importante mencionar que no desenvolvimento dessa Campanha Nacional, como no POSE, os professores receberam treinamento para realizarem as ações de saúde ocular propostas.

No exame dos resultados, observou-se que a maioria dos professores $(55,9 \%)$ declarou ter identificado alunos que apresentavam dificuldades visuais, corroborando os achados de Alves, Temporini e Kara-José que, em trabalho recente ${ }^{(17)}$, reportaram que o professor foi quem percebeu a dificuldade visual do escolar em 70,6\% dos casos estudados, quando pesquisaram sobre o atendimento oftalmológico de escolares do sistema público de ensino no município de São Paulo ${ }^{(17)}$.

Na tabela 3, em relação às ações realizadas pelos professores, verificou-se que $84,2 \%$ proveram orientações ao escolar e $63,2 \%$ à família, para o encaminhamento do problema. É altamente recomendável a parceria escola-família visando a saúde visual e o rendimento escolar dos alunos. Por isso, os pais podem colaborar, sendo informados sobre os cuidados com a visão para observar o comportamento visual da criança e encaminhar os filhos para exame oftalmológico ${ }^{(8)}$.

Verificou-se que apenas 26,3\% dos professores declararam ter orientado o escolar a procurar o oftalmologista (Tabela 3). De acordo com o relato da maioria dos professores, esse baixo índice justifica-se pelo fato de não se sentirem aptos para esse encaminhamento. Neste sentido, vale ressaltar que o professor pode detectar alunos que apresentem dificuldades visuais no desempenho das tarefas escolares e realizar encaminhamentos para os serviços oftalmológicos para buscar soluções. No entanto, é importante mencionar que não é de sua competência a realização de diagnósticos.

Os resultados apresentados na tabela 3 evidenciam ações que não apresentam expressividade em termos quantitativos.
Esse fato evidencia a necessidade do preparo dos professores, direcionado à detecção de dificuldades visuais e às medidas para minimizar ou solucionar tais problemas.

Por isso, é reconhecida a necessidade de programas de preparação dos professores e de toda comunidade escolar, para a disseminação do conhecimento e a participação em programas de prevenção de problemas oftalmológicos em escolares ${ }^{(18)}$.

Em relação à saúde ocular, as ações do professor na escola referem-se ao que ele sabe, acredita e deseja. A incapacidade para tomar resoluções mais efetivas está relacionada à ausência do conhecimento ${ }^{(2)}$. No presente estudo, observou-se interesse dos professores em esclarecer dúvidas sobre aspectos da dificuldade visual. Muitos professores declararam que a partir do momento em que preencheram o questionário foram despertados para a observação de sinais e sintomas indicativos de dificuldades visuais e poderiam futuramente identificar alunos que apresentavam problemas visuais.

\section{CONCLUSÃO}

Os professores apresentaram conhecimento insuficiente quanto à saúde ocular e, portanto, as ações desenvolvidas não foram completas e abrangentes. Sugere-se a implantação de um programa de saúde ocular em todo o sistema público de ensino, visando desenvolver ações de prevenção da incapacidade visual, promoção e recuperação da saúde ocular.

Sugere-se a implantação de um programa de saúde ocular em todo o sistema público de ensino, com abrangência sobre todas as séries escolares do ensino fundamental, visando desenvolver ações de prevenção da incapacidade visual, promoção e recuperação da saúde ocular. E, dentro desse programa, inserir a saúde ocular dentro dos currículos escolares, como era feito na época do Plano de Oftalmologia Sanitária Escolar - POSE.

\section{ABSTRACT}

Purpose: 1) To check knowledge and actions developed by teachers of elementary schools working with low vision students, regarding signals and symptoms evidencing the students' visual difficulties: 2) To collect information useful for the design of preventing actions regarding eye health in school. Methods: A survey was applied to teachers of elementary schools in 23 public schools in the country of Campinas / SP, with low vision students, during the academic year of 1999. A self-applied questionnaire was used as tool for data obtention. Results: Of 84 teachers, 68 (81\%) answered the questionnaire. The average teaching experience was 20.8 years. Most of those teachers $(92.6 \%)$ did not report any previous specific training in visual deficiency. As concerns the recognition of signs and symptoms, most of the teachers indicated blackboard reading difficulty $(94.1 \%)$, followed by headache $(89.7 \%)$ and placement of subjects too 
close to the eyes (88.2\%). A little over half of the teachers (55.9\%) identified students showing visual difficulties. As regards developed actions $84.2 \%$ of the teachers declared to have oriented the students and $63.2 \%$ oriented parents and relatives and also informed the school about the fact. Only $26.3 \%$ oriented the student to look for an ophthalmologist. Conclusion: Teachers did not show adequate knowledge about eye health and thus the developed actions were not comprehensive. Therefore, in order to develop actions regarding the prevention of visual impairment and to promote the enhancement of eye health, the implementation of a program aiming at the care of eye health in the public school is suggested.

Keywords: Vision, low; Vision disorders; School health; Eye health; Knowledge attitudes and practice; Health education; Students; Visual acuity; School health services

\section{REFERÊNCIAS}

1. Alves MR, Kara-José N. O olho e a visão: o que fazer pela saúde ocular das nossas crianças. Rio de Janeiro: Vozes; 1996.

2. Armond JE, Temporini ER. Crenças sobre saúde ocular entre professores do sistema público de ensino no Município de São Paulo, SP, Brasil. Rev Saúde Pública 2000;34:9-14.

3. Thylefors B, Négrel AD, Pararajasegaram R, Dadzie KY. Global data on blindness. Bull the World Health Organ 1995;73:115-21.
4. Kara-José N, Almeida GV, Arieta CEL, Araújo JS, Becgara SJ, Oliveira PR. Causas de deficiência visual em crianças. Bol Oficina Sanit Panam 1984;47: 405-13.

5. Alves MR, Kara-José N. Manual de orientação ao professor. Campanha Nacional de Reabilitação Visual. CBO, MECE, FNDE. São Paulo; 2000.

6. Kara-José N, Saba HC, Cartocci AA, Braga AC, Shimoda GA, Guidi HC, et al. Conhecimentos e práticas em saúde ocular de 1000 pessoas da cidade de Campinas, São Paulo. Arq Bras Oftalmol 1985;48:160-4.

7. Macchiaverni Filho N, Kara-José N, Rueda G, Pereira VL, Costa MN, Rangel $\mathrm{FF}$, et al. Levantamento oftalmológico em escolares da primeira a quarta séries do primeiro grau na cidade de Paulínia, São Paulo. Arq Bras Oftalmol 1979;42:289-94.

8. Temporini ER. Aspectos do plano de oftalmologia sanitária escolar do Estado de São Paulo. São Paulo. Rev Saúde Pública 1982;16:243-69.

9. Gilbert C, Foster A. Childhood blindness in the context of VISION 2020 the right to sight. Bull World Health Organ 2001;79:227-32.

10. Novaes HMD, Zucolotto SMC. A saúde do escolar. In: Almeida MJ, Camargo ES, Collares CAL, Moyses MAA. Fracasso escolar - uma questão médica? São Paulo. Cortez-Cedes, 1985. p.17-29. (Caderno CEDES, 15).

11. Kara-José N, Temporini ER. Avaliação dos critérios de triagem visual de escolares de primeira série do primeiro grau. Rev Saúde Pública 1980;14:205-14

12. Oliveira RCS, Kara-José N, Sampaio MW. Entendendo a baixa visão: orientação aos professores. Brasília: MEC/SEESP; 2000

13. Temporini ER. Prevenção de problemas visuais de escolares: conduta de professores do sistema de ensino do Estado de São Paulo, Brasil. Rev Bras Saúde Esc 1990;18:259-62.

14. Piovesan A, Temporini ER. Pesquisa exploratória: procedimento metodológico para o estudo de fatores humanos no campo da saúde pública. Rev Saúde Pública 1995;29:318-25.

15. Gasparetto MERF. Visão subnormal em escolas públicas: conhecimentos, opinião e conduta de professores e diretores do ensino fundamental [tese]. Campinas: Universidade Estadual de Campinas; 2001.

16. Temporini ER. Percepção de professores do sistema de ensino do Estado de São Paulo sobre o seu preparo em saúde escolar. Rev Saúde Pública 1988; 22:411-21.

17. Alves MR, Temporini ER, Kara-José N. Atendimento oftalmológico de escolares do sistema público de ensino no município de São Paulo - aspectos médico-sociais. Arq Bras Oftalmol 2000;63:359-63.

18. Focesi E. Uma nova visão de saúde escolar em saúde na escola. Rev Bras Saúde Esc 1992;2:19-21.

\section{Simpósio da Associação Paranaense de Oftalmologia}

\section{4 a 26 de Junho de 2004 \\ Bourbon Hotel - Curitiba - PR}

INFORMAÇÕES: JDE ComunicaçãoeEventos

Tels.: (1 1 ) 287-9378/289-4301

Fax: (1 1) 288-8157

E-mail:jdecomev@uol.com.br 